\title{
Selective transvenous embolization of dural carotid-cavernous sinus fistulas with preservation of sylvian venous outflow
}

\section{Report of three cases}

\author{
Mitsugu Nakamura, M.D., Norihiko Tamaki, M.D., Tetsuro Kawaguchi, M.D., and Shigekiyo \\ Fujita, M.D.
}

Department of Neurosurgery, Kobe University School of Medicine, Kobe, Japan; and Department of Neurosurgery, Hyogo Brain and Heart Center at Himeji, Himeji, Japan

A transvenous embolization technique that spares normal cerebral venous drainage is described. Of 26 dural carotid-cavernous fistulas treated by the authors, in three cases the affected cavernous sinus was supplied by not only the shunt flow but also the sylvian venous drainage. Two patients presented with an abducent nerve palsy and one with an oculomotor nerve palsy in whom selective transvenous embolization of the fistulous portions of the affected cavernous sinus was achieved while preserving of the sylvian venous outflow. Posttransvenous embolization angiograms showed complete occlusion of the fistula in one patient and only small residual shunts in the other two; one underwent subsequent transarterial embolization, whereas the other was followed without additional treatment. The patients' symptoms disappeared between 1 and 2 months posttreatment. Follow-up angiograms revealed that the remnant shunt had disappeared and that the sylvian venous pathway had been preserved. The authors conclude that although the condition is uncommon it is important to recognize that a dural carotid-cavernous sinus fistula, which receives significant sylvian venous outflow, can be treated successfully by selective transvenous embolization of the fistulous compartments in an affected sinus.

Key Words * dural arteriovenous fistula * cavernous sinus * transvenous embolization * endovascular therapy * sylvian vein

In the treatment of dural arteriovenous fistulas (AVFs) involving the cavernous sinus, transvenous occlusion of the affected cavernous sinus has been found to effect a better cure than other treatment options. $[3,7,8,10,12,13]$ Because transvenous embolization ordinarily results in complete occlusion of the cavernous sinus, few reports have discussed treatment of dural carotid-cavernous sinus fistulas (CCFs) that receive not only the shunt blood flow but also sylvian venous drainage. When the sylvian venous outflow significantly contributes to the normal venous drainage, problems can arise with occluded portions of the sinus that receive normal venous drainage from parts of the brain. We report the cases of three patients in whom AVFs in the cavernous sinus are treated. Although the sylvian vein 
entered the affected cavernous sinus, selective transvenous embolization of the fistulous parts of the cavernous sinus was successfully performed with preservation of the sylvian venous outflow pathway.

\section{CASE REPORTS}

Since 1992, we have treated 26 patients of dural CCFs in three of whom the affected cavernous sinus received not only arteriovenous shunt flow but also the normal sylvian venous outflow. The three patients were all women, ranging in age from 57 to 59 years. Two patients presented with abducent nerve palsy and one with an oculomotor nerve palsy. Digital subtraction angiograms showed that the fistulas drained into the superior and medial parts of the sinus and that the sylvian venous outflow entered the ipsilateral sinus at the anterolateral portion.

Selective transvenous embolization of the fistulous parts of the affected cavernous sinus and preservation of the sylvian venous outflow was attempted in all three cases. After placement of No. 5 French sheaths in the right femoral vein and left femoral artery, No. 5 French catheters were then introduced into the common carotid artery and the right inferior petrosal sinus, and a Fas-Tracker 18 microcatheter (Target Therapeutics, Fremont, CA) was introduced into the affected cavernous sinus. After detailed examination of arteriograms and venograms, the fistulous portions of the affected cavernous sinus were occluded with interlocking detachable coils ([IDCs]; Target Therapeutics) in two cases and Guglielmi detachable coils ([GDCs]; Target Therapeutics) in one. Follow-up angiograms were obtained 6 and 12 months after postembolization.

\section{Case 1}

This 59-year-old woman presented with horizontal diplopia. On admission, neurological examination revealed a right-sided abducent nerve palsy. Right external and internal carotid angiograms demonstrated a dural AVF involving the right cavernous sinus (Fig. 1 upper left). The fistulous flow drained into the right inferior petrosal sinus and contralateral cavernous sinus, and the normal superficial sylvian vein drainage entered the affected cavernous sinus and then flowed into the pterygoid plexus (Fig. 1 upper right). The microcatheter was easily passed through the medial and superior parts of the affected sinus into its anterolateral portion. Venography, which was performed via the microcatheter with its tip placed at the anterolateral portion of the cavernous sinus, demonstrated flow of contrast material both to the inferior petrosal sinus, into which the arteriovenous shunt drained, and to the pterygoid plexus, in which the normal venous drainage entered (Fig. 1 center). We placed an initial IDC just posterior to the superficial sylvian venous pathway and confirmed its patency by angiography before coil detachment. Selective embolization of the superior and medial parts of the cavernous sinus was performed with a total of 12 IDCs. Postembolization right common carotid artery angiograms demonstrated closure of the arteriovenous shunts (Fig 1 lower left) and preservation of the superficial sylvian vein pathway (Fig 1 lower right). The patient's right-sided abducent nerve palsy resolved 1 month postembolization; no lesions were seen on follow-up angiograms, and no recurrence was observed during a follow-up period of 18 months. 

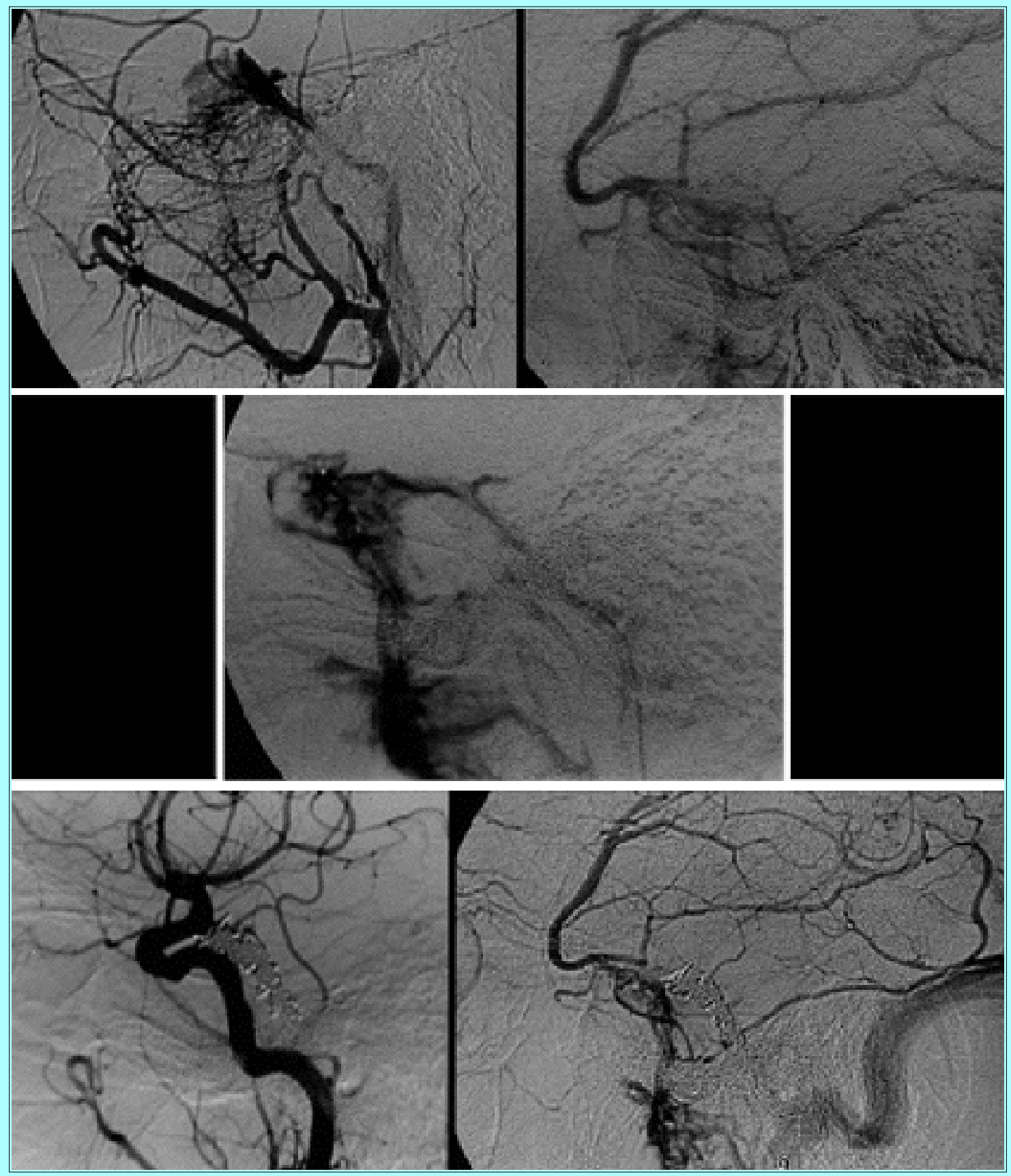

Fig. 1. Case 1. Angiographic study. Upper: Preembolization right external (left) and internal (right) carotid artery angiograms demonstrating a dural CCF draining into the ipsilateral inferior petrosal sinus and the superficial sylvian venous outflow draining into the affected cavernous sinus, with the flow continuing to the pterygoid plexus. Center: Intraoperative venogram through a microcatheter demonstrating the contrast material draining into the inferior petrosal sinus as well as the pterygoid plexus. Lower Left and Right: Postembolization right common carotid artery angiograms showing closure of the fistulas and preservation of the sylvian venous outflow.

\section{Case 2}

This 57-year-old woman presented with a right-sided abducent nerve palsy. Angiography demonstrated a dural CCF draining into the right inferior petrosal sinus and contralateral cavernous sinuses (Fig. 2 left). The normal superficial and deep sylvian veins entered the affected sinus and then drained into the pterygoid plexus (Fig. 2 right). 


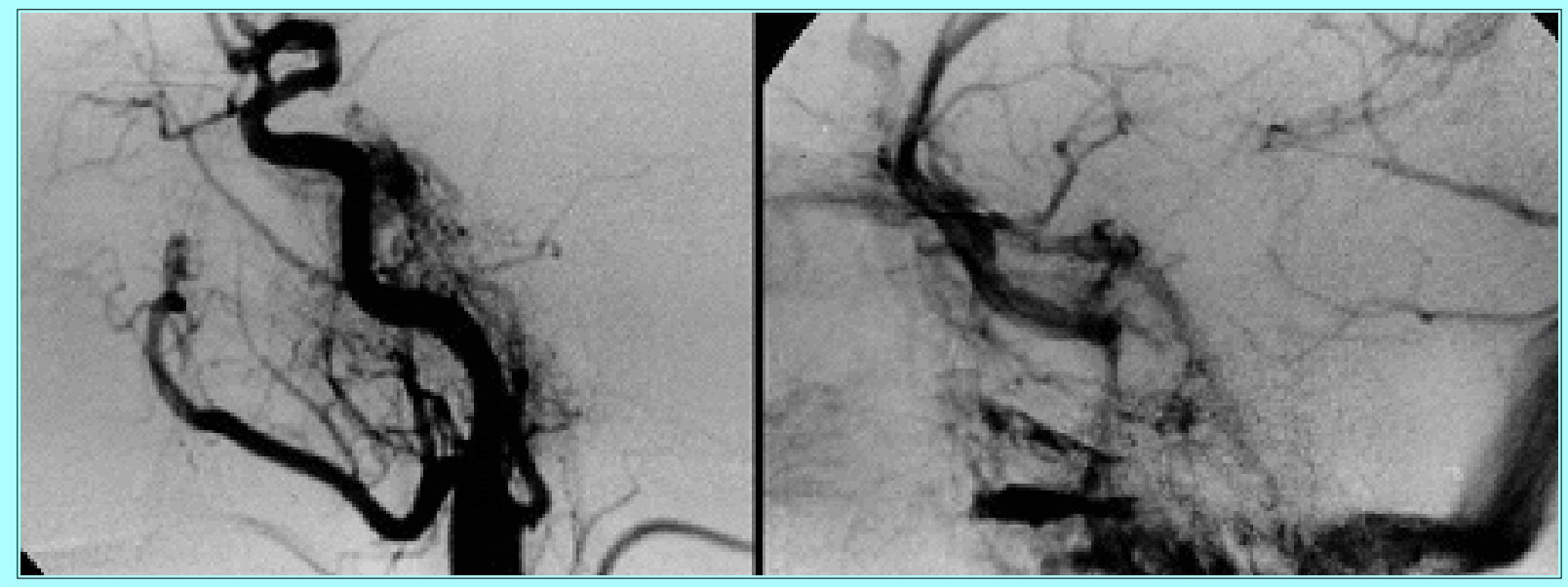

Fig. 2. Case 2. Preembolization right common carotid artery angiograms demonstrating a dural CCF that drains into the inferior petrosal sinus [left], and the sylvian venous outflow, that drains into the affected cavernous sinus [right].

As in Case 1, the microcatheter was easily passed through the fistulous part of the cavernous sinus into the normal sylvian venous pathway. We placed an initial IDC just posterior to the deep sylvian venous pathway, but its drainage path changed to the superficial sylvian venous during the embolization procedure. Selective embolization of the fistulous parts of the cavernous sinus was performed using a total of 30 IDCs. It was extremely difficult to achieve complete occlusion in the fistulous parts of the sinus while preserving the part receiving the sylvian venous outflow. However, postembolization angiography demonstrated preservation of the superficial sylvian venous pathway and residual arteriovenous shunt draining into the contralateral cavernous sinus. The remnant shunt had disappeared on follow-up angiograms at 12 months (Fig. 3 left), while the sylvian venous outflow was preserved (Fig. 3 right). The patient's right-sided abducent nerve palsy was resolved by 2 months postembolization.

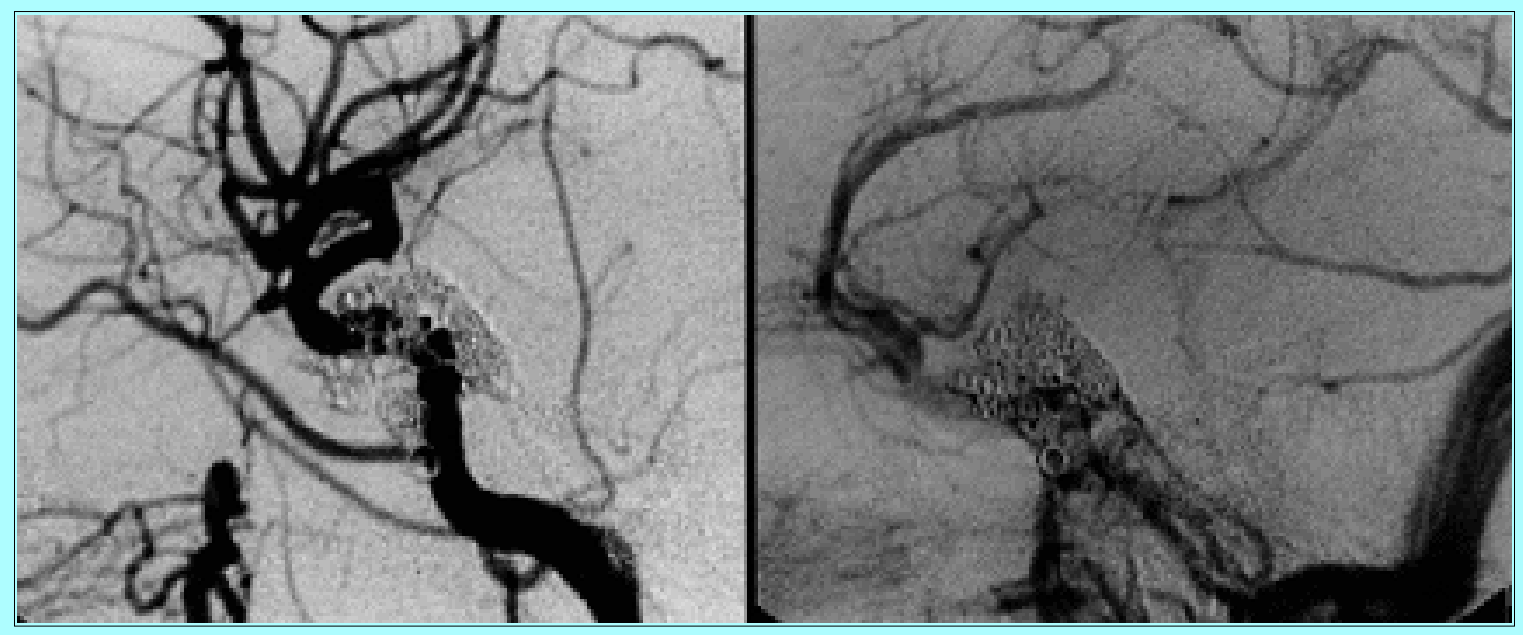

Fig. 3. Case 2. Left and Right: Follow-up common carotid angiograms at 12 months demonstrating disappearance of the shunt and preservation of the superficial sylvian venous pathway.

\section{Case 3}

This 57-year-old woman presented with a right-sided oculomotor nerve palsy. Angiography demonstrated a dural CCF that drained into the right inferior petrosal and contralateral cavernous sinuses. The normal superficial sylvian vein drainage entered the affected cavernous sinus and then flowed, 
together with the fistulous drainage, into the contralateral cavernous sinus. In contrast with the other two cases, the pterygoid plexus had not sufficiently developed in this patient. The GDC that transiently occluded the inferior intercavernous sinus was retrieved because it significantly retarded wash-out of the contrast material in the superficial sylvian vein and cavernous sinus. After occluding the superior and medial parts of the sinus with a total of $20 \mathrm{GDCs}$, subsequent transarterial embolization was performed via the ipsilateral middle meningeal artery and the maxillary artery with platinum coils for a remnant shunt. Postembolization angiograms demonstrated closure of the fistula and preservation of the superficial sylvian venous pathway that drained into the contralateral cavernous sinus. The patient's right-sided oculomotor nerve palsy was resolved by 1 month postembolization, and follow-up angiograms at 6 months showed no fistulous lesions.

\section{DISCUSSION}

\section{Endovascular Treatment of Dural CCF}

Several treatment options, including compression therapy,[6] transarterial embolization of feeding arteries,[15] transvenous occlusion of the involved sinus,[3,7,8,10,12,13] conventional radiotherapy,[2] and stereotactic radiosurgery,[9] have been used for the treatment of dural AVFs in the cavernous sinus, and transvenous embolization of the affected sinus frequently has been the treatment of choice. Introduction of thrombogenic material directly into the involved dural sinus was first described by Mullan.[10] Hosobuchi[8] described surgical placement of copper wire into the affected cavernous sinus followed by electrocoagulation to occlude the sinus. Vascular access to the cavernous sinus has been reported, including surgical exposure of the angular or superior ophthalmic vein, $[3,8,12,13]$ the transethmoidal-transsphenoidal surgical approach,[1] and the transfemoral approach through the ipsilateral inferior petrosal sinus, the angular vein, and the contralateral cavernous sinus.[13] Copper wires, silk sutures, platinum microcoils, IDCs, and n-butyl-cyanoacrylate have been used as embolic materials.

Transvenous occlusion performed in the affected cavernous sinus usually can include the entire sinus without risk, because in most cases the affected cavernous sinus does not contribute to normal venous drainage. In rare cases, however, dural AVFs involve a cavernous sinus that significantly participates in normal venous drainage. In our clinical experience, these uncommon cases occurred in $12 \%$ of dural CCFs. In such cases complete occlusion of the cavernous sinus can result in venous infarction by which outflow is obstructed; however, few reports have discussed a treatment option for such cases. Watanabe, et al.[16] have reported a patient with a dural CCF, in whom the cavernous sinus received the normal cortical drainage from the insular vein. In this patient, postembolization magnetic resonance imaging demonstrated cerebral infarction due to congestion in the area of the posterior insular vein because the anticipated drainage of the insular vein via the uncal vein following embolization had not occurred. Uncertainty about whether to occlude the whole sinus or to occlude the affected portions selectively makes it difficult to select a treatment option in these uncommon cases. Whole-sinus embolization could eliminate the fistula but has the disadvantage of potential venous infarction. Selective occlusion could result in sparing the normal venous pathway but has the potential disadvantage of changing the fistulous flow into the normal brain.

\section{Modified Transvenous Embolization Technique for Dural AVF}

Our group[11] has reported elsewhere that, for superior sagittal and transverse fistulas, embolization of the venous compartment of the fistula located within the sinus wall, prior to the sinus lumen, can 
preserve or restore the impaired sinus function. This technique, however, could not be used to treat dural CCFs because the mural venous compartments of the fistula did not dilate enough to allow the microcatheter to be advanced. The cavernous sinus has unique structural features, that are different from the other venous sinuses. The cavernous sinus can be divided into several anatomical parts by the internal carotid artery running inside the sinus. The posteromedial part of the cavernous sinus connects the superior and the inferior petrosal sinuses. The anterolateral part connects the superficial and deep sylvian vein, the superior and inferior ophthalmic vein, and the pterygoid plexus. Although there are individual differences in terms of the connections and the collateral pathways among these sinuses and veins, detailed angiographic examination of the fistulas and normal venous outflow make it possible to embolize the fistulous portions selectively and, simultaneously, to preserve the normal venous outflow. In our three cases the arteriovenous shunts entered the superior and medial portions of the cavernous sinus, and flowed from the sylvian vein entered the anterolateral portion of the sinus. We could not determine by preembolization angiography whether or not the fistulous portions and the sylvian vein outflow communicated functionally within the cavernous sinus. During the embolization procedure, a microcatheter could be easily passed through the fistulous portion into the normal venous pathway inside the sinus. Venograms obtained via the microcatheter demonstrated flow of contrast material both into the fistulous drainage pathway and into the normal venous outflow pathway. Accordingly, we postulated that there was no complete structural separation of the two compartments but that trabeculae, partial sinus thrombosis, or other factors appeared to separate the two compartments hemodynamically within the affected cavernous sinus.

Great care was taken with the initial coil positioning, including pretreatment examination of detailed angiograms because the coil should completely occlude a connection between the normal and fistulous parts inside the sinus to prevent retrograde cortical drainage. An anteroposterior view provided particularly important information for initial coil placement. After detachment of the initial coil, the affected portions of the cavernous sinus were packed as densely as possible. When we placed the last coil in the inferior portion of the cavernous sinus, we reconfirmed patency of the sylvian venous pathway on angiography. Yoshimura, et al.,[17] and Terada et al.,[14] also used IDCs and described the use of varied coil lengths as well as detachment and retrieval mechanisms in the embolization of dural arteriovenous shunts. We believe such retrievable coils as IDCs and GDCs[4,5] are suitable for difficult embolization procedures. On the other hand, this modified transvenous technique for dural CCFs has several disadvantages; for example, it is important to preserve the normal venous outflow pathway, but achieving complete occlusion of the fistulous compartments of the cavernous sinus is quite difficult. When a residual shunt is present, careful observation of the patient's symptoms and evaluation of follow-up angiograms are necessary or the procedure should be combined with transarterial embolization. Although the spared venous pathway maintained patency on follow-up angiograms in our three patients, late thrombosis of the venous outlet might result from platinum coils placed beside the normal venous pathway. If the recurrent fistula appears in the spared portion, the presence of coils could prevent recatheterization into the fistulous site. Transarterial embolization and/or radiosurgery should be recommended in such cases.

In conclusion, it is clinically important to recognize the uncommon case of a dural carotid-cavernous sinus fistula that also receives the normal cerebral venous outflow. Transvenous embolization can be used to treat these cases. This technique requires careful planning based on preoperative angiography and intraoperative venography and involves the placement of retrievable coils. 


\section{References}

1. Barker F G II, Ogilvy CS, Chin JK, et al: Transethmoidal transsphenoidal approach for embolization of a carotid-cavernous fistula. Case report. J Neurosurg 81:921-923, 1994

2. Bitoh S, Hasegawa H, Fujiwara M, et al: Irradiation of spontaneous carotid-cavernous fistulas. Surg Neurol 17:282-286, 1982

3. Courtheoux P, Labbe D, Hamel C, et al: Treatment of bilateral spontaneous dural carotid-cavernous fistulas by coils and sclerotherapy. Case report. J Neurosurg 66:468-470, 1987

4. Guglielmi G, Vinuela F, Dion J, et al: Electrothrombosis of saccular aneurysms via endovascular approach. Part 2: Preliminary clinical experience. J Neurosurg 75:8-14, 1991

5. Guglielmi G, Vinuela F, Sepetka I, et al: Electrothrombosis of saccular aneurysms via endovascular approach. Part 1: Electrochemical basis, technique, and experimental results. J Neurosurg 75:1-7, 1991

6. Halbach VV, Higashida RT, Hieshima GB, et al: Dural fistulas involving the cavernous sinus: results of treatment in 30 patients. Radiology 163:437-442, 1987

7. Halbach VV, Higashida RT, Hieshima GB, et al: Transvenous embolization of dural fistulas involving the cavernous sinus. AJNR 10:377-383, 1989

8. Hosobuchi Y: Electrothrombosis of carotid-cavernous fistula. J Neurosurg 42:76-85, 1975

9. Link MJ, Coffey RJ, Nichols DA, et al: The role of radiosurgery and particulate embolization in the treatment of dural arteriovenous fistulas. J Neurosurg 84:804-809, 1996

10. Mullan S: Treatment of carotid-cavernous fistulas by cavernous sinus occlusion. J Neurosurg 50:131-144, 1979

11. Nakamura M, Tamaki N, Hara Y, et al: Two unusual cases of multiple dural arteriovenous fistulas. Neurosurgery 41:288-293, 1997

12. Takahashi A, Yoshimoto T, Kawakami K, et al: Transvenous copper wire insertion for dural arteriovenous malformations of cavernous sinus. J Neurosurg 70:751-754, 1989

13. Teng MM, Guo WY, Huang CI, et al: Occlusion of arteriovenous malformations of the cavernous sinus via the superior ophthalmic vein. AJNR 9:539-546, 1988

14. Terada T, Kinoshita Y, Yokote H, et al: Clinical use of mechanical detachable coils for dural arteriovenous fistula. AJNR 17:1343-1348, 1996

15. Vinuela F, Fox AJ, Debrun GM, et al: Spontaneous carotid-cavernous fistulas: clinical, radiological, and therapeutic considerations. Experience with 20 cases. J Neurosurg 60:976-984, 1984

16. Watanabe A, Hirano K, Suzuki Y, et al: Venous congestion of the insular cortex after transvenous coil embolization of a dural carotid-cavernous fistula in Taki W, Picard L, Kikuchi H (eds): Advances in Interventional Neuroradiology and Intravascular Neurosurgery. Amsterdam; Elsevier, 1996, pp $271-274$

17. Yoshimura S, Hashimoto N, Kazekawa K, et al: Embolization of dural arteriovenous fistulas with 
Manuscript received August 17, 1998.

Accepted in final form September 25, 1998.

Address reprint requests to: Mitsugu Nakamura, M.D., Department of Neurosurgery, Kobe University School of Medicine, 7-5-1 Kusunoki-cho, Chuoh-ku, Kobe 650, Japan. email: naka-m@med.kobe-u.ac.jp. 